\title{
Name Entity Recognizer for Odia using Conditional Random Fields
}

\author{
R. C. Balabantaray ${ }^{1 *}$, S. K. Lenka ${ }^{2}$ and D. Sahoo ${ }^{3}$ \\ 'Asst Prof., CLIA Lab, Department of Computer Science, IIIT, Bhubaneswar, Odisha, India; rakeshbray@gmail.com \\ 2 Project Fellow, CLIA Lab, IIIT, Bhubaneswar, Odisha, India; sanjaya.lenka@gmail.com \\ ${ }^{3}$ Research Project Fellow, CLIA Lab, IIIT, Bhubaneswar, Odisha, India; deepsahoo@gmail.com
}

\begin{abstract}
Name Entity Recognition (NER) is a process of information extraction that seeks to locate atomic elements in text and classify them into predefined categories such as the name of persons, organizations, locations, expressions of times, quantities, monetary values, percentages, etc. In this paper, we present an Odia Named Entity Recognizer which will be very useful in searching of information about not only tourism but also all general domains. Here, we follow the method of conditional random field. This is a machine learning technique based on linguistic rules of Name Entities (NE). It handles nested tagging of name entities with a hierarchical tag set containing forty four attributes (level one), thirty eight attributes (level two) and thirty five attributes (level three) in tag set. We have experimented building Conditional Random Field (CRF) models by training the noun phrases of the training data and it gives encouraging results.
\end{abstract}

Keywords: Odia Tourism Corpus, Name Entity Recognition, Precision, Recall, F-measure.

\section{Introduction}

The sole aim of information extraction by machine is to crystallize the references which can convey highest amount of referent of the text. Naturally, unusual and much more referent can be connoted by a Name Entity. So, the name entity is an important linguistic element in a particular text. The significance of name entity in a text is all the information denoted by a sentences and paragraphs is directed to and based on Name entity. In other words the name entity in sentence and paragraphs generates text and also extracts text. This paper proposes a computational mechanism to recognize the name entity in odia text.

As we know Odia is an official language of Odisha and it is the first language of more than thirty million people and second and third language of many people of India. Odisha and odia language is bound by three distinct language areas that are Telugu in the south and south-west, Chhattisgarhi (Hindi) in the west and Bengali in the north. It is also significance to say that the internal linguistic composition of Odisha state consists of many tribal groups, who linguistically belong to two distinct language families-Dravidian and Munda. So, the name entities in these languages have been nativised through borrowing in Odia. This language has history of more than one thousand and ten years. It is also rich in literature for which it deserves reconisation of classical language.

There is no morphological and punctuation mark helping us to recognise Name entity in Odia. This element can be easily rounded out in discourse of this language. So, it can be argued that NE in this language is based on semantic field of this language. The name entity in this language is in some extent different from that of other language and in some extent it is same with that of other language. It is different in the sense that is related with tourism, geographical diversity, culture and history of this state. For example, this state has so many tourist places as temple, hillside, waterfall, lake, sea beach, riverbank, Science Park and science

* Corresponding author:

R. C. Balabantaray (rakeshbray@gmail.com) 
museum and so many religious pendals. Some of them are well known in world like Jaganarth temple and lord jaganath, Konark suntemple, lingaraj temple, Madhabananda temple, Mahandi, Chilika lake, Puri cart festival, Samrat Ashok, Kaling war, Khandagiri, Udayagiri, and Dayanadi. There are some NEs which are seen in this language as well as in other languages, for example, Baudha dharam, odisi dance, and name of the people.

All name entities are proper noun but they can function as noun, adjective and adverb phrase in sentence. This makes a single name entity can appear as different phrase in different places of a sentence. When a name entity is used, it can be inflected but in many cases name entity does not take overt inflectional form but it can have covert inflectional form. There are some name entities like date, money which occur in a specific pattern.

Name entity in this language can be single word or more than single word but within the phrase of name entity containing more than one words there can be a covert grammatical relation which is called compositionality of construction.

\section{Conditional Random Fields (CRF)}

Conditional Random Fields (CRF) [11] is a machine learning technique. CRF overcomes the difficulties faced in other machine learning techniques like Hidden Markov Model (HMM) [9] and Maximum Entropy Markov Model (MEMM) [5]. HMM does not allow the words in the input sentence to show dependency among each other. MEMM shows a label bias problem because of its stochastic state transition nature. CRF overcomes these problems and performs better than the other two. HMM, MEMM and CRF are suited for sequence labelling task. But only MEMM and CRF allows linguistic rules or conditions to be incorporated into machine learning algorithm.

Lafferty, McCallum and Pereira define a CRF on observations $X$ and random variables $Y$ as follows:

Let $G=(V, E)$ be a graph such that $\boldsymbol{Y}=\left(\boldsymbol{Y}_{v}\right)_{v \in V}$, so that $Y$ is indexed by the vertices of $G$. Then $(X, Y)$ is a conditional random field when the random variables $\boldsymbol{Y}_{v}$ conditioned on $X$, obey the Markov property with respect to the graph: $p\left(\boldsymbol{Y}_{v} \mid \boldsymbol{X}, \boldsymbol{Y}_{w}, w \neq v\right)=p\left(\boldsymbol{Y}_{v} \mid \boldsymbol{X}, \boldsymbol{Y}_{w}, w \sim v\right)$, where means $\omega \sim v$ that $\omega$ and $v$ are neighbors in $G$.

What this means is that a CRF is a undirected graphical model whose nodes can be divided into exactly two disjoint sets $X$ and $Y$, the observed and output variables, respectively; the conditional distribution $p(Y \mid X)$ is then modeled.
For general graphs, the problem of exact inference in CRFs is intractable. The inference problem for a CRF is basically the same as for an MRF and the same arguments hold. However there exist special cases for which exact inference is feasible:

- If the graph is a chain or a tree, message passing algorithms yield exact solutions. The algorithms used in these cases are analogous to the forward-backward and Viterbi algorithm for the case of HMMs.

- If the CRF only contains pair-wise potentials and the energy is submodular, combinatorial $\mathrm{min}$ cut/max flow algorithms yield exact solutions.

If exact inference is impossible, several algorithms can be used to obtain approximate solutions. These include:

- Loopy belief propagation

- Alpha expansion

- Mean field inference

- Linear programming relaxations

Learning the parameters $\theta$ is usually done by maximum likelihood learning for $p\left(Y_{i} \mid X_{i} ; \theta\right)$. If all nodes have exponential family distributions and all nodes are observed during training, this optimization is convex. It can be solved for example using gradient descent algorithms, or Quasi-Newton methods such as the L-BFGS algorithm. On the other hand, if some variables are unobserved, the inference problem has to be solved for these variables. Exact inference is intractable in general graphs, so approximations have to be used.

CRFs can be extended into higher order models by making each $Y_{i}$ dependent on a fixed number $o$ of previous variables $Y_{i-\sigma^{\prime}}, \ldots, Y_{i-1}$. Training and inference are only practical for small values of $o$ (such as $o \leq 5)$, since their computational cost increases exponentially with $o$.

\section{Methodology}

Many techniques have been implemented in Indian and European languages for NER. Some of them are rule based system [7], which makes use of dictionary and patterns of named entities, Decision trees [8], Hidden Morkov Model (HMM) [4], Maximum Entropy Morkov Model (MEMM) [3], Conditional Random Fields (CRF) [2] etc. In short, the approaches can be classified as rule-based approach, machine learning approach or hybrid approach. For Indian languages, many techniques have been experimented by different people. MEMM system for Hindi NER [6] gave an 
average F1 measure of 71.9 for a tagset of four named entity tags. These tag sets are great help in NER but these need modification and revision. Because there are some grammatical categories that can overlap and cannot be tagged by this tag set. The problems with these tagset are it is based on limited number of grammatical categories which cannot tag sub categories of a super ordinate category.

NER has been done generically and also domain specific where a finer tag set is needed to describe the named entities in a domain. We have developed a hierarchical tagset consisting of 117 tags for tourism and general domain. We have used Conditional Random Fields, a machine learning approach to sequence labelling task, which includes NER.

We have used a hierarchical tagset containing 44 (level one), 38 (level two) and 35(level three) tags. The tag set is designed considering the optimal grammatical categories in noun phrase.

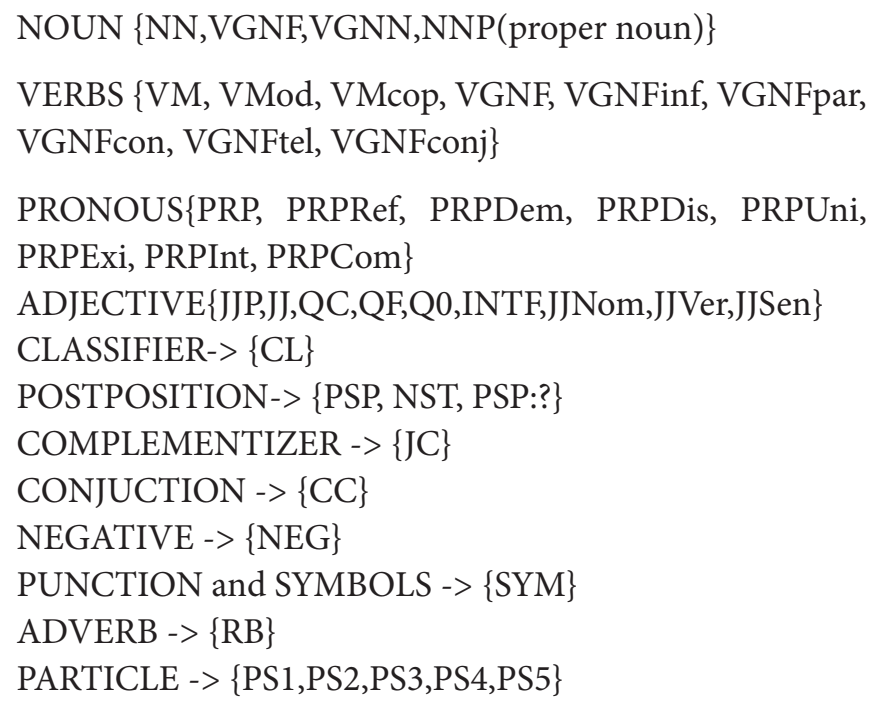

\begin{tabular}{|c|c|c|c|c|}
\hline 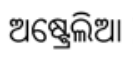 & NNP & B-NNP & 1 & B-PLACE \\
\hline ধાสิ6ฉ & $\mathrm{NN}$ & I-NNP & 0 & o \\
\hline ๑|ฉ૪ & NNP & B-NNP & 1 & B-PLACE \\
\hline - & SYM & I-NNP & 0 & o \\
\hline થ6ब్తి̄లు & NNP & I-NNP & 1 & B-PLACE \\
\hline$\checkmark Q$ & QC & B-NNP & 0 & o \\
\hline ฉิธฮิณ & $\mathrm{JJ}$ & I-NNP & 0 & o \\
\hline Я૫ા८6૨ & NN & I-NNP & 0 & o \\
\hline ๑|ฉ૪ & NNP & B-NNP & 1 & B-PLACE \\
\hline 4 & QC & B-NNP & 0 & o \\
\hline 386ロ56 & SQNN & I-NNP & 0 & o \\
\hline 6வ|প|ঋ্রি & $\mathrm{JJ}$ & B-VP & 0 & o \\
\hline
\end{tabular}

\begin{tabular}{|c|c|c|c|}
\hline ลิติ & $\mathrm{NN}$ & I-NNP & 0 \\
\hline இ|बm & NN & I-VP & 0 \\
\hline ติลิญิ & VM & I-VP & 0 \\
\hline 1 & SYM & I-VP & 0 \\
\hline
\end{tabular}

The Sample training Tagged Data is given below for reference:

Test data will also be presented in way similar to how we presented the training data. Test data is processed for Morph analysis, POS [1] and NP chunking [10]. Here also, the same set of attributes and feature templates are used. Now, the test data is tagged with each of the CRF models built for three levels of hierarchy.

\begin{tabular}{|c|c|c|}
\hline ฉฉด & $\mathrm{NN}$ & I-NNP \\
\hline ฮูฉ & INTF & B-NNP \\
\hline ধুঞ্ম & $\mathrm{JJ}$ & I-NNP \\
\hline 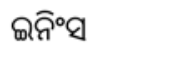 & $\mathrm{NN}$ & I-NNP \\
\hline 3 & $\mathrm{CC}$ & I-NNP \\
\hline শપપાઘ6ઠૃ & $\mathrm{NN}$ & B-NNP \\
\hline Я6థ9 & $\mathrm{NNP}$ & I-NNP \\
\hline$\widehat{\natural}^{\circ}$ & NNP & I-NNP \\
\hline 6્dા৪1૯ & NNP & I-NNP \\
\hline 6శા & JJ & B-NNP \\
\hline ও७४ & $\mathrm{NN}$ & I-NNP \\
\hline இ৭। & $\mathrm{NN}$ & I-NNP \\
\hline ఆ|৫৪৫ & NNP & B-NNP \\
\hline ลิตณฑ్ & $\mathrm{NN}$ & I-NNP \\
\hline gા. & PS1 & B-VP \\
\hline થูลิธิธ & $\mathrm{JJ}$ & I-NNP \\
\hline 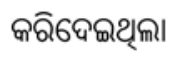 & VM & B-VP \\
\hline I & SYM & I-VP \\
\hline ওઘฉฉ & $\mathrm{JJ}$ & B-NNP \\
\hline 6ளிษЯ & NNP & I-NNP \\
\hline எЯ1ฉष্লি & NNP & I-NNP \\
\hline 92 & QC & B-NNP \\
\hline
\end{tabular}

\section{Experiment and Results}

We have created $277 \mathrm{~KB}$ (About 9630 tagged words) word corpus in Odia in tourism and general domain. POS 
tagging, NP chunking and named entity annotation are done manually on the corpus. We conducted the standard 10 fold experiment, in each folder there will be training data and test data. Then in each folder a learning model is created based on the training data. Generally training data and the test data are in the ratio of $80 \%$ to $20 \%$ of the total data.

Here we present the Precision, Recall and F-measure for each set of the conducted 10 fold experiment.

$\begin{array}{lrrl}\text { Precision } & & \text { Recall } & \text { F-measure } \\ \text { Set0 } & \mathbf{0 . 3 0} & \mathbf{0 . 3 2} & \mathbf{0 . 3 1} \text { (Lowest) } \\ \text { Set1 } & 0.66 & 0.64 & 0.65 \\ \text { Set2 } & \mathbf{0 . 8 1} & \mathbf{0 . 7 8} & \mathbf{0 . 7 9} \text { (Highest) } \\ \text { Set3 } & 0.60 & 0.55 & 0.58 \\ \text { Set4 } & 0.73 & 0.66 & 0.69 \\ \text { Set5 } & 0.54 & 0.45 & 0.49 \\ \text { Set6 } & 0.66 & 0.61 & 0.64 \\ \text { Set7 } & 0.74 & 0.68 & 0.71 \\ \text { Set8 } & 0.59 & 0.56 & 0.58 \\ \text { Set9 } & 0.67 & 0.65 & 0.66\end{array}$

\section{Conclusion}

In this paper, we have developed a NER system using CRF with the help of a NE tagged Odia Corpus. We have taken standard name entity tagset that was used for annotation of this corpus. We have considered features such as POS, IOB2 format, information about the surrounding words and their tags. We found from the experiments that Conditional Random Fields is also well suited for Name Entity recognition task in Indian languages. Once we will have more number of tagged files in the Odia corpus for training the system we are hopeful of getting still better result than this.

\section{References}

1. Arulmozhi P, Sobha L et al. (2004). Parts of speech tagger for Tamil, Symposium on Indian Morphology, Phonology \& Language Engineering, March 19-21, IIT Kharagpur, 55-57.

2. McCallum A, and Li W (2003). Early results for named entity recognition with conditional random fields, feature induction and web-enhanced lexicons, CONLL '03 Proceedings of the seventh conference on Natural language learning at HLT-NAACL 2003, vol 4.

3. Borthwick A, Sterling J et al. (1998). Description of the MENE named entity system, Proceedings of the Seventh Machine Understanding Conference (MUC-7).

4. Bikel D M (1997). Nymble: a high-performance learning name-finder, Proceedings of the Fifth Conference on Applied Natural Language Processing, 194-201.

5. Berger A, Della Pietra S et al. (1996). A maximum entropy approach to natural language processing, Computational Linguistics, vol 22(1), 39-71.

6. Kumar N, Bhattacharyya P (2006). Named entity recognition in Hindi using MEMM, the proceedings of Technical Report, IIT Bombay.

7. Krupka G R, and Hausman K (1998). Iso Quest Inc: Description of the NetOwl text extraction system as used for MUC-7, Proceedings of Seventh Machine Understanding Conference (MUC 7).

8. Karkaletsis V, Pailouras G et al. (2000). Learning decision trees for named-entity recognition and classification, Proceedings of the ECAI Workshop on Machine Learning for Information Extraction.

9. Rabiner L R (1989). A tutorial on hidden markov models and selected applications in speech recognition, Proceedings of the IEEE, vol 77(2), 257-286.

10. Sobha L, Ram V S R (2006). Noun Phrase Chunking in Tamil, Proceedings of the MSPIL-06, IIT Bombay, 194-198.

11. John Lafferty, Andrew McCallum, et al. (2001). Conditional random fields: probabilistic models for segmenting and labeling sequence data, Proceedings of the Eighteenth International Conference on Machine Learning (ICML2001), 282-289. 